Volume No. 1, Issue No. 2 (April- June), pp. 10-25

\title{
Self-Enabling Vehicular Agent using Cloud and Massive Data
}

\author{
Shahid Naseem, Muhammad Shabbir and Fahad Ahmad
}

\begin{abstract}
Since last some decades, the autonomous technology in the vehicles was used to help the drivers to voyage effortlessly along the highways and to avoid road accidents. In this duration, a number of high-end vehicles was built-in electronic secureness mechanism, adaptive voyage mechanism, lane departure warnings and city safety system. Approximately, $95 \%$ of the road accidents were caused by the wrong behavior, careless, focus less and tiredness of the drivers. Even with the attractiveness of recent traffic control applications, a lack of dynamic information about roads and weather conditions was more than an infuriation. For this purpose, we developed a self-enabling vehicular agent in which cloud and Massive data is used. The cloud and massive data enabled the agent to see around corners or even miles down the road and to drive itself more carefully. The parameters enabled the agent to keep the driver informed on the road conditions ahead. The vehicular agent would be enable to process the appropriate data from this massive data, send it via cloud to forecasts of the traffic situation, the road conditions, the cars are ahead of it and the weather in the real time.
\end{abstract}

Keywords: cloud, massive data, self-enabling, memories, vehicular agent.

\section{INTRODUCTION}

Transport was a complex activity that was used to connect different locations i.e. proximate, well-connected and displaying high level of access in many urban areas with flow. The flow between these locations meant people or goods and the number of vehicles to carry these people and goods. In the past, the autonomous vehicles relied on

Shahid Naseem, Ph.D Scholar NCBA\&E, Lahore, Pakistan.

Asst. Prof. UCEST, Lahore Leads University Lahore, Pakistan. Email:- shahid.naseem@gmail.com

Muhammad Shabbir, MS (CE), UET, Taxila, Pakistan.

Asst. Prof. UCEST, Lahore Leads University Lahore, Pakistan.

Fahad Ahamd, Ph.D Scholar NCBA\&E, Lahore, Pakistan.

IT Manager Services Institute of Medical Sciences, Lahore, Pakistan. 
erudite systems and multiple sensors to analyze and adapt to a rapidly changing environment. In these vehicles, the availability of appropriate information about events, people and objects had not been possible. In these vehicles, autonomous features were increasingly more complex and in-vehicle test will no longer be sufficient. In these vehicles, test creates constraints to repeatability and comparability as well as large costs and time. Vast amount of data was required to be stored and used during development of these vehicles. Now a days, in the digital universe, information about photographs, movies and surveillance video feeds, data was produced in the form of digital contents and processed to create phone call metadata using some medium such as sensors. This information represented an overwhelming amount of data and a denotation portion relates to vehicle sensor outputs [1].

Last some decades, location-sensing technologies are being used for tracking people, vehicles upto few centimeters more precisely. In locationsensing technologies, the synthesis of cloud data is used to track the vehicles and its flow record. In these technologies, the data about the location and routes is intrinsically in nature but it is very difficult to anonymous effectively, because it can create risks for misuse as potential operations of individuals and their behavior.

The exploitation of massive data was increasingly important and upsetting changes in different sectors such as transport. The urban planning division was using different techniques i.e. physical data regarding the inferred use of municipal space by citizens, realtime data regarding vehicle operation and contiguous environment to analyze the massive data and to minimize potentially dangerous conflicts. Mobility-related data could resolve policy challenges related to congestion and safety because failure to account for changing nature of data collection, sue and access could lead to negative outcomes such as unintended and unwanted erosion of privacy rights [2].

The massive data was not linked with a single technological changes rather, it was the confluence of new data collection mechanism based on ubiquitous digital devices. The massive data was also enhanced storage capacity and computer power as well as enhanced sensing and communication technologies. These communication technologies were used on real-time 
data transmission of massive amount of data. These communication technologies were also used to address well-defined questions and to resolve specific tasks [3]. Transport-related data patterns could open up new avenue for misuse of data and potential manipulation of individuals and their behavior. No mechanism was available for addressing autonomous vehicles complexity, security and safety, validation and verification. The failure in the autonomous vehicular system, would take surely complicate the regulatory tasks.

\section{MATERIALS \& METHODS}

A cloud and massive data based selfenabling vehicular agent is used to improve the reliability and security to reduce the vehicular operational cost. It is also used to develop, validate and verify mixed integrity applications with real-time requirements. One of the main tasks for highly and fully automatic vehicles using the cloud is an information carrier for the vehicle. Because automatic vehicles are needed to know what is next on the road, i.e. the road is free or not, there is any construction or not. The cloud and Massive data enables the agent to avoid accidents, to minimize the road traffic, keep connected it with the world around it. But not all of data is relevant to the agent. The agent is able to process the pertinent data and make accurate predictions of the road ahead, pulling what is relevant from this massive data, packing it together into a secure protocol, and sending it via the cloud to a massive backend that can make sense of the traffic situation, the road conditions, the cars on the road and the weather all in the real time.

\section{PRIVACY OF MASSIVE DATA IN VEHICULAR AGENT}

In vehicular agent, sensors are used to enhance road safety. In case of location data, data protection strategies are sheathing behind new approaches of data collection and uses. In this agent, a privacy technique to control and allocate rights to data controller because failing to secure of an important data may result in a regulatory repercussion against the collection and processing of location data. This action could creel revolution and bound the social and economic benefits [4].

\section{HUMAN-MACHINE INTERACTION}

A self-enabling vehicular agent cannot control issue excellently, although, its 
use can restricted to areas, where the vehicles can assertively handle driving complexity.

\section{AUTOMATION IN CLOUD- BASED VEHICLE ARCHITECTURE}

The automation of conventional vehicles is unlikely to change vehicle market dynamics. Users can buy and own such automatic vehicle, using automatic vehicles in certain situations like driving on motorways, parking a car, or handling stop-and-go-traffic. individuals travel and has an impact on industries such as public transport and taxis. In automatic cloud-based vehicular architecture, different rules are considered for testing, licensing and

Operation of autonomous vehicle prototypes. The autonomous vehicular agent can regulate vehicles to ensure safety and prevent of market failures. The automation will regulate flexibility seems desirable for instance allowing circumscribed uses such as low speed urban operation or motorway
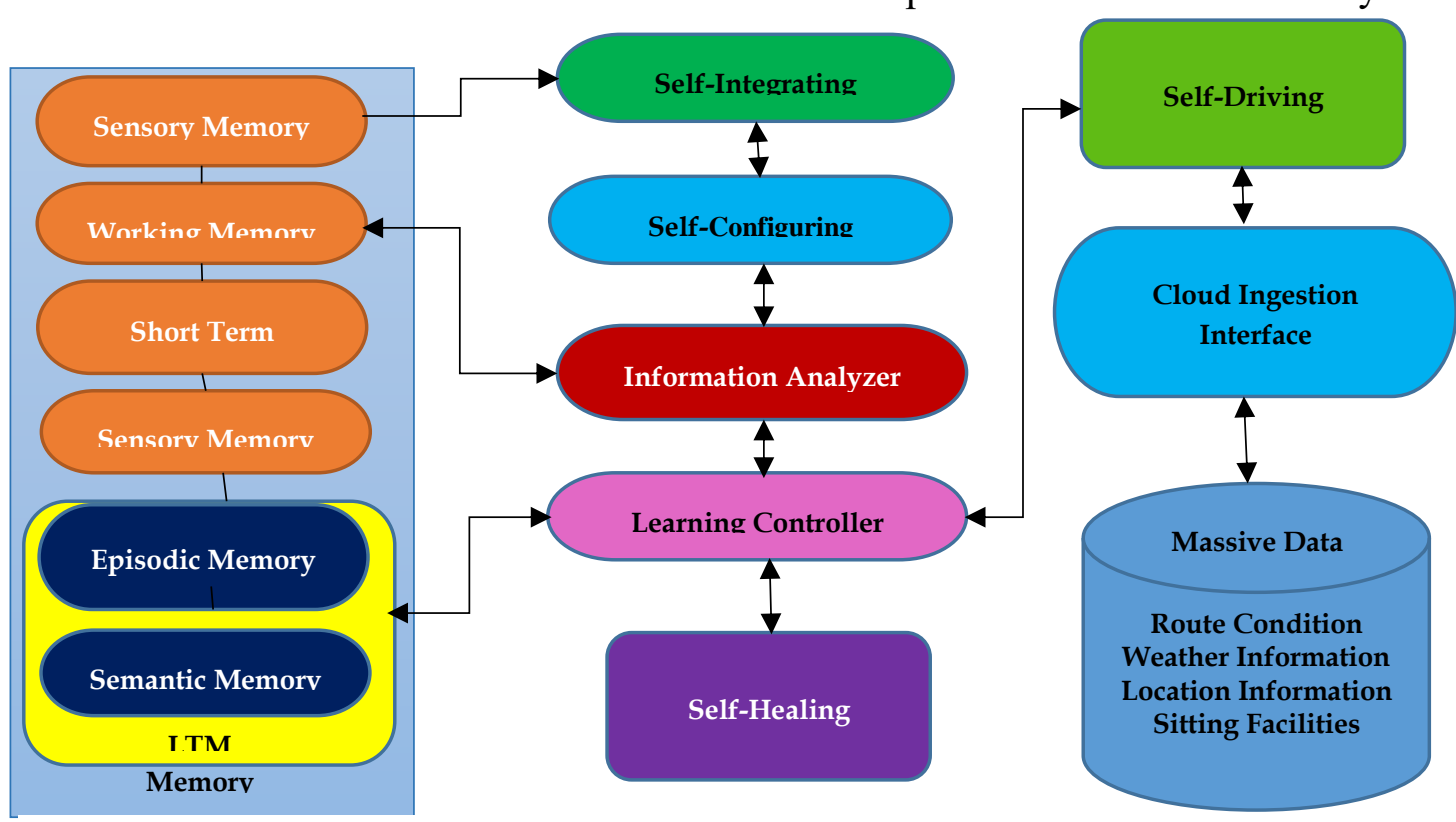

Figure 1: Self-Enabling Vehicular Agent

Automated vehicular agent is much capable to handle interruption. It can be deployed in fleet-wide systems that would fundamentally reshape shifted from humans to machines, an 
insurance regime is implemented. In average [5].

the architecture, regulate and develop different activity plans to minimize legacy risks. The purpose of regulating and developing activity plans is to limit the physical risk vehicles through monitoring, over the air updates and even virtual recalls presented by legacy. In Autonomous Vehicular agent, sensors are used to reduce the legacy risks.

\subsection{Sensory Memory:}

Sensory memory is used to receive information about the events occurred in the external environment. It has unlimited storage memory and it holds only the information which has to process further for decision making as well as store in the long-term memory. It stores only the meaningful information that is not yet stored in it. It stores the information about the events occurred for a specific period of time that may be less than a second. If it holds the information more than a second, the information may lost and furthermore, it can't be maintained any kind of cognitive activity. In sensory memory, when a $3 \times 4$ array of letters are processed then after an interval of only 0-1 seconds the sensory memory does not remember all the letters, it can only hold 5-6 o events information on

\subsection{Working Memory:}

Working memory is responsible for processing the run-time activity. It consists of several elements such as phonological loop, visual-spatial sketchpad, and central executive and episodic buffer. Phonological loop is responsible for acoustic practice such as reiterating a mobile number again and again is responsible for reprocesses visual descriptions of an impetus, essential supervision is responsible to control cognizant informationprocessing and episodic cushion to secondary memory.

\subsection{Short-Term Memory (STM):}

Sort-term memory contains the information that is to be reprocessed. By means of consideration, some information is transferred to Primary Memory or Short-Term Memory (STM) after it has received some degree of processing i.e. after it has been subject to feature detection, pattern recognition, and directed attention. Typically, primary memory is thought of as storing an acoustic representation of the information i.e. what the name or description of an object or event sounds like. Primary 
memory has a limited capacity. It can contain only about 7 plus items.

Information is sustained in STM by means of reprocess. If it is not reprocesses the information either lost, or more may be changed with the new information. The storage capacity of STM is often confirmed by different tests. But the capacity of STM can be increased by scattering items together in some meaningful way. STM is the pathway to long-term memory. This implies that the system can't have longterm memory if it doesn't have shortterm memory.

\subsection{Long-Term Memory (LTM):}

LTM is used to hold the information about the events occurred in the external environment for long time e.g. minimum 20 to 30 minutes, a day, a month, a year for number of years. It holds the information permanently and can be reprocessed or can be used whenever is needed in future. It receives the information from shortterm memory through working memory. It is a permanent memory and information in it can't be deleted or altered. It can be deleted or altered whenever the user is required to delete it. Its capacity is unlimited.

\subsection{Episodic Memory:}

Episodic memory is autobiographical memory and is concerned with anybody's personal experiences. It describes the specific events as well as includes a description of the episodic content i.e. time and place in which the events occurred as well as a reference to the self as the agent or experiencer of these events. The reference to the self may also include additional information about the person's cognitive, emotional and motivational state at the time of the event [6].

\subsection{Semantic Memory:}

Semantic memory concerns with one's context-free, knowledge. Information stored in semantic makes no reference to the context in which it is acquired, and no reference self as agent or experiencer of events. Knowledge stored in semantic memory is categorical, including information about subset-superset relations, similarity relations and categoryattribute relations. Semantic memory is often portrayed as a network, with nodes representing individual concepts 
and links representing the semantic or conceptual relations between them.

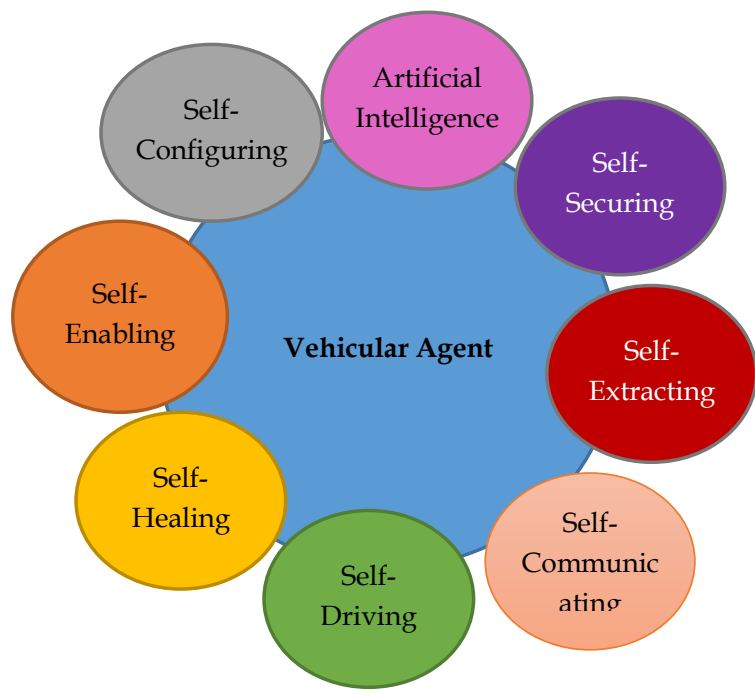

Figure 2: Self-Enabling Vehicular Agent's functions

The main objective for developing a self-enabling vehicular agent is to collecting all pertinent massive data, whether it comes from a component, the passengers, or the transportation infrastructure and whether it relates to the operation of the vehicle or any other aspect of the transportation experience. As it receives through cloud, not all of the data that is generated would need to be collected and managed. For example, self-enabling agent requires the capture of far more data than the personalization of in-car entrainment choices to the driver's tastes. The agent can be used the abstracted data from massive data for real-time traffic alerts, real-time public transportation alerts etc. All the data and models used by the vehicular agent can be available over the cloud [7].

\subsection{Self-Integrating:}

Self-integrating module is used to integrate the collected data. It will collect and use information from other concerning traffic, mobility, weather and other events associated with moving around such as details about driving conditions, as well as sensor-

based and location-based information. Because of the quantity and variety of low-level data through sensors to gather information about the surrounding environment is appropriate necessary for significant amount of data fusion and integration to be done on board.

\subsection{Self-Configuring:}

Self-configuring module is used to receive the cloud data from selfintegrating module and convert into digital format for using in the computer to provide the desired vehicle experience, for example, personal preferences on configuring controls, 
driving habits or medical information about the driver or its usual occupants.

\subsection{Self-Learning:}

As the self-enabling vehicular agent will have cognitive capabilities to learn the behavior of the driver and occupants, the agent itself and the surrounding environment to continually optimize and advise. As the agent learns more about the driver and occupants, it will be able to expand its advice to other mobility services options.

\subsection{Self-Healing:}

In the self-enabling vehicular agent, self-healing module is responsible to fix and optimize itself based on certain events or situations without intervention or inconvenience. The analytical capability of the agent will help to identify and locate issues, schedule fixes and even will help other vehicles with similar problems with minimal impact to the driver.

\subsection{Self-Driving:}

In the framework, the learning module will analyze the data, if it finds the autonomous vehicle require any kind of healing, then it will send the signal to the self-healing module, otherwise, it will send the signal for self-driving module. As the vehicular agent as become highly automated with some areas of limited autonomous functions in controlled environments.

\subsection{Artificial Intelligence:}

In the autonomous vehicular agent, the artificial intelligence is used to help the agent to recognize patterns and learn from the behavior of other vehicles on the road. The artificial intelligence can include the abilities to direct the vehicular agent to any facility on the road such as gas station near to it on the road or stay location etc.

\subsection{Self-Communicating:}

Self-communicating module will connect the autonomous vehicle with other vehicles and the infrastructure around it to share information and solution.

\subsection{Self-Extracting:}

Self-extracting module is used to capture the massive data from cloud about the weather, road condition, filling pump near to agent on the road, construction information on real-time and to abstract on the relevant information to be used for safety driving on the road. 


\subsection{Self-Securing Data:}

In view of risk of hackers accessing the data of autonomous vehicles, data security is one of the massive issue in developing self-enabling vehicular agent. To safeguard against data, in addition to cyber security solutions, the security module is responsible to look into data encryption solutions.

Self-enabling agent enables autonomous vehicle to communicate through the cloud infrastructure. In this agent, a significant step to derive a global standard for vehicle-to-cloud data is made which in turn brings the promise of automated vehicles closer to reality. In the agent, the sensors gather the connected cars data that exists in multiple different formats in the external environment and send it to the cloud for processing and analysis. The agent has the capability to detect and process changes in the real world as they happen such as accidents on the road, road construction, weather condition etc. For example, if a car around the next corner hits the brakes because there's an obstruction, that information could be used to signal to the drivers behind to slow down ahead of time, resulting in smoother, more efficient journeys and a lower risk of accidents. But that can only work if all cars are autonomous working [8].

Self-enabling vehicular agent interface is defined for exchanging information between the in-vehicle sensors and a dedicated cloud as well as between clouds will enable broad access, delivery and processing of vehicle sensor data, enable easy exchange of vehicle sensor data between all modules and finally enable enriched location based services which are key for mobility services as well as for automated driving. In the architecture, vehicle data exchange will enable the development of smart mobility services such as real-time traffic, weather and parking spaces in the short term while holding the promise to power selfenabling vehicles with critical high accuracy real-time mapping capabilities in the future.

\section{RESULTS AND DISCUSSION}

The autonomous vehicular systems are erudite systems that are based on multiple sensors such as cameras, Wi$\mathrm{Fi}$, radar etc. to capture, analyze and adapt in the rapidly changing environment. But in these systems, the availability of appropriate information about the 
Table 1: Number of death and injuries on the road crashes in Pakistan between 2010-2016

\begin{tabular}{|c|c|c|c|c|}
\hline$\dot{\Xi}$ & $\begin{array}{l}\overline{\bar{w}} \\
\tilde{\Xi}\end{array}$ & $\stackrel{D}{0}$ & & 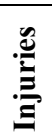 \\
\hline & $\begin{array}{l}\text { Bus fell in } \\
\text { River }\end{array}$ & $\begin{array}{l}\text { Haramos } \\
\text { h Peak }\end{array}$ & 12 & 7 \\
\hline 2011 & $\begin{array}{l}\text { Bus vs. Oil } \\
\text { Tanker }\end{array}$ & & 32 & 9 \\
\hline 2012 & $\begin{array}{l}\text { Two Buses } \\
\text { Accident }\end{array}$ & & 17 & 19 \\
\hline 2013 & $\begin{array}{l}\text { Different } \\
\text { oad } \\
\text { ccidents, } \\
\text { us + Truck } \\
\text { us+Trailer,B } \\
\text { +car }\end{array}$ & Kohistan & 81 & 50 \\
\hline 2014 & \begin{tabular}{|l|} 
School \\
Van+Truck \\
, Bus +Car, \\
two buses \\
Two \\
tankers, \\
bus \\
+tracktor \\
Trailer, \\
Coach+Tru \\
ck
\end{tabular} & $\begin{array}{l}\text { Sindh, } \\
\text { Baluchist } \\
\text { an,Hub, } \\
\text { Sukkar, } \\
\text { Sindh }\end{array}$ & 168 & 18 \\
\hline 2015 & $\begin{array}{l}\text { Bus+Oil } \\
\text { Tanker }\end{array}$ & Sindh & 62 & \\
\hline
\end{tabular}

Events occurred in the external environment have not been possible. In these systems, the autonomous features are increasing more complex with the increasing of events information. Vast amount of data is required to be store and use during the development of these systems. To overcome these vehicular problems, we have developed a self-enabling vehicular agent that has the capability to reduce the traveling time, increase the security and safety level using the cloud and massive data. The massive data in the database contains the information about the road condition, construction on the road, location of a petrol pump or gas pump on the road near the vehicle, location of sitting place or restaurant on the road. The cloud is used to communicate the massive data to vehicular agent to reach at its desire place with safe and sound. In the autonomous vehicular agent, we have developed different levels of mechanization checking the control and operations of the agent on the basis of automation, integration, and configuration and healing.

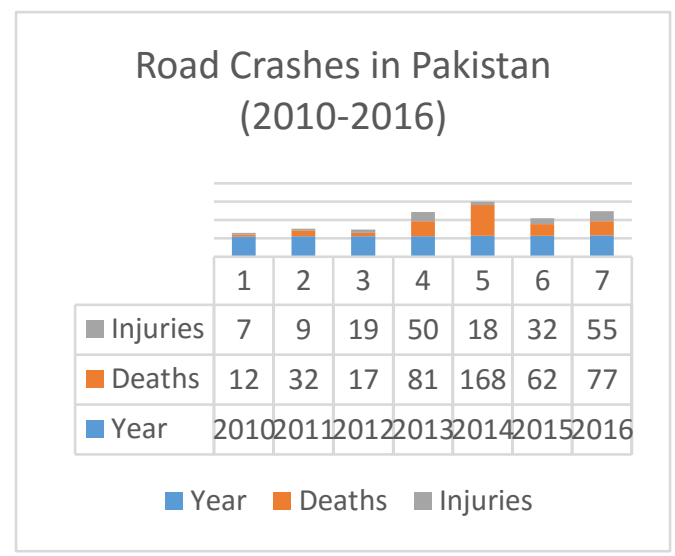

Figure 3: Road crashes in

Figure 3 indicates the number of road crashes occurred in Pakistan during the period 2010 to 2016 in different cities, provinces and the number of deaths and injuries. From this figure we can see 
that there were 168 persons were lost their lives and 18 persons were injured in the year 2014 in different cities of Sindh, Baluchistan provinces. Similarly, maximum number of injuries were recorded in year 2016 due to road accidents in Sheikhupura and D.I. Khan Districts. The graph represents the bar chart of the number of year in blue color, number of death in yellow color and number of injuries in gray color.

Table.2: Levels of Mechanization of Vehicular Agent

\begin{tabular}{|c|c|c|}
\hline & Control & Operations \\
\hline 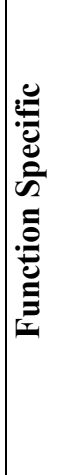 & 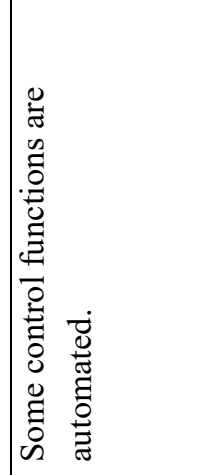 & 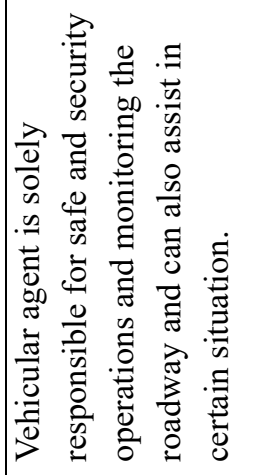 \\
\hline 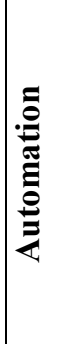 & 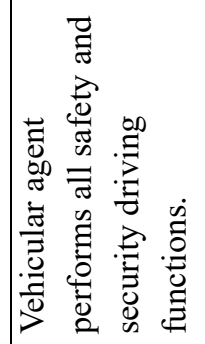 & 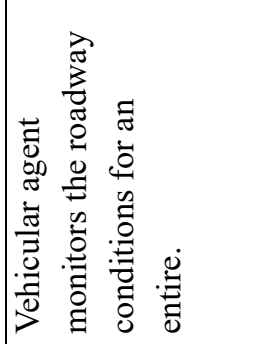 \\
\hline
\end{tabular}

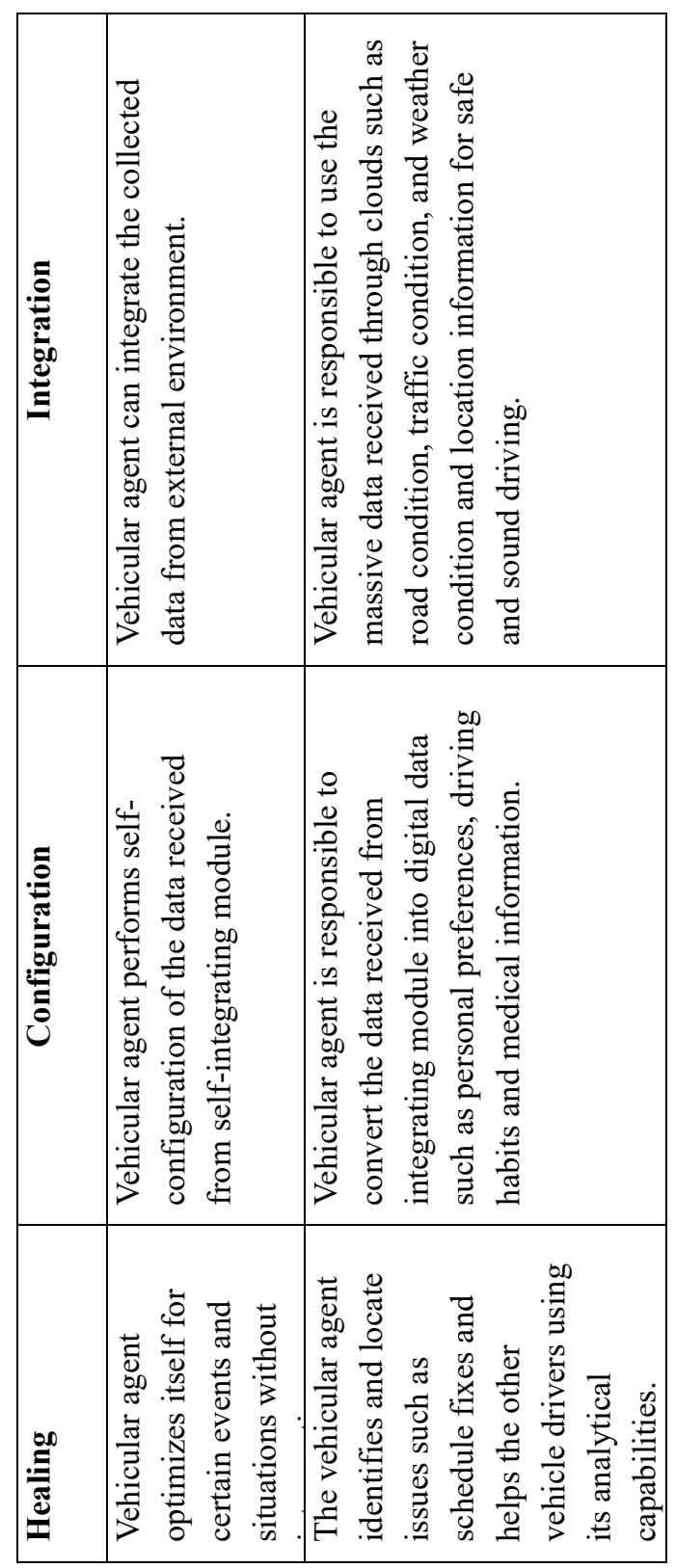




\begin{tabular}{|c|c|c|}
\hline 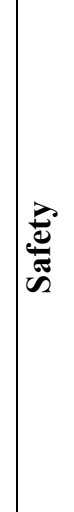 & 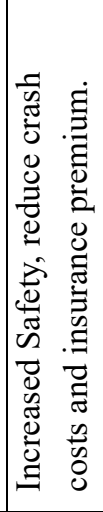 & 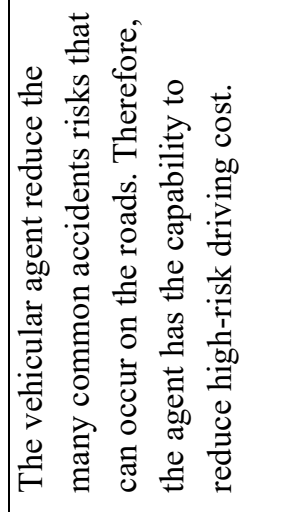 \\
\hline 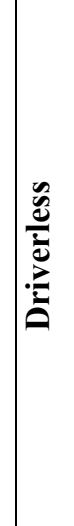 & 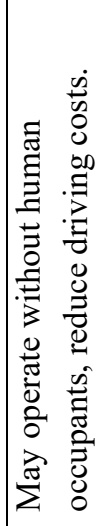 & 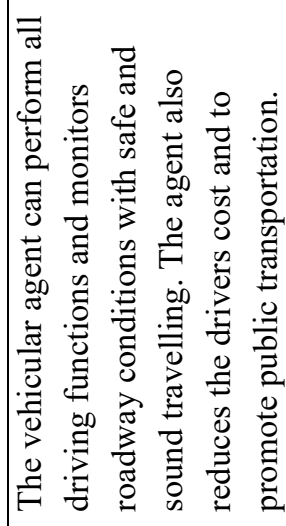 \\
\hline 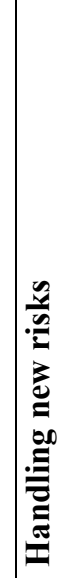 & 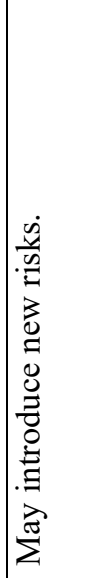 & 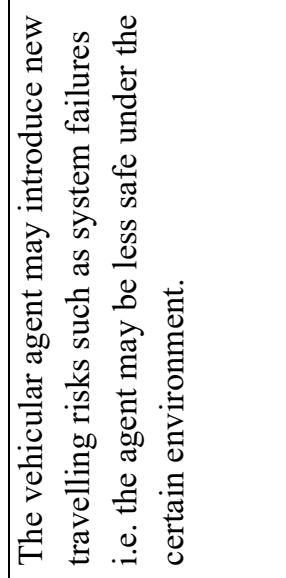 \\
\hline
\end{tabular}

In this table, we have defined two parts of each rule i.e. control and operation. Each rule has some control that the vehicular agent has. In the control column, we have just defined that what is the purpose of each rule and in operation column, we have defined the detail functionality of each rule.

Table 3: personally vehicles, driverless vehicles and self-enabling vehicles per mile cost (\$)

\begin{tabular}{|c|c|c|c|c|}
\hline \multirow[t]{2}{*}{$\stackrel{\mathscr{e}}{\ddot{z}}$} & \multicolumn{2}{|c|}{ 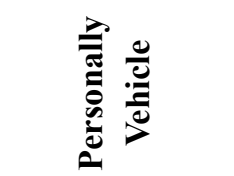 } & \multirow[t]{2}{*}{ 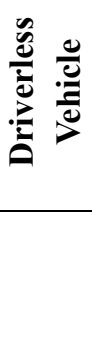 } & \multirow[t]{2}{*}{ 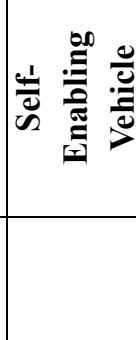 } \\
\hline & 音 & 高 & & \\
\hline 5 & & & & 0.07 \\
\hline 10 & 0.2 & 0.2 & 0.22 & \\
\hline 15 & & & & 0.08 \\
\hline 20 & & & & \\
\hline 30 & & & 0.19 & \\
\hline 40 & & & 0.39 & 0.41 \\
\hline 45 & 0.55 & & & \\
\hline 50 & & & & \\
\hline 60 & 0.59 & & & \\
\hline 75 & 0.75 & & & \\
\hline
\end{tabular}

Table 2 indicates the different level of mechanization that are required by a vehicular agent to become autonomous. 
Figure 4 indicates the comparative better analysis.

analysis between the personally vehicles, driverless vehicles and selfenabling vehicles. In this table we have taken 80 miles distance for comparison purpose and measure different cost per mile for these four types of vehicles. After comparison, we have found that the self-enabling vehicle is more

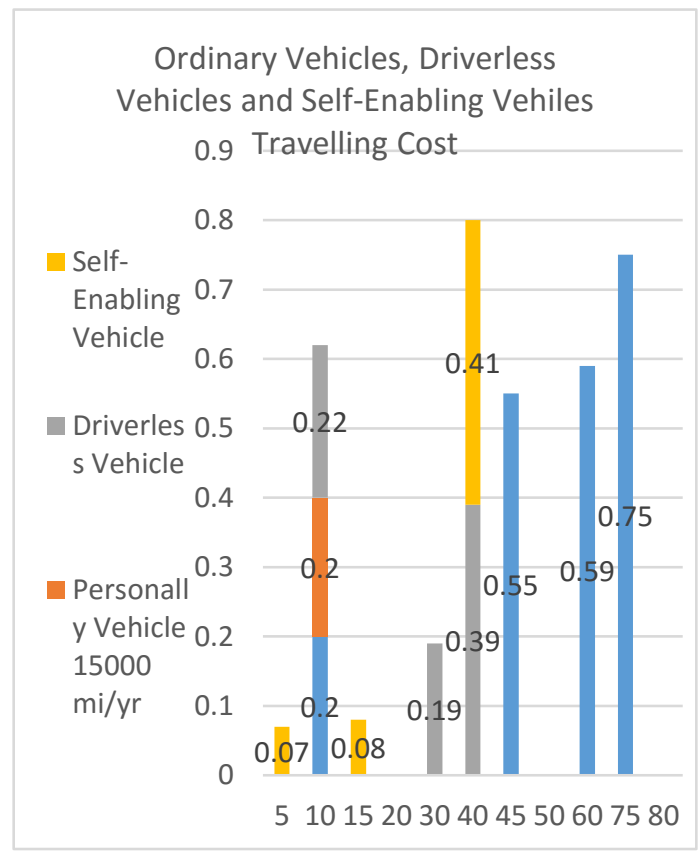

Figure 4: Ordinary vehicles, Driverless, Selfenabling

suitable for fuel consumption wise and cost per mile wise because it reduces the driver cost as well as repair maintenance cost. In the below, we have presented this comparative analysis in the bar cart graph wise for

\section{SIMULATION}

We have simulated our agent's performance using Fuzzy Interface logic operators and IF-Then rules. We have used Mamdani type FIS for simulating our inputs. FIS is the

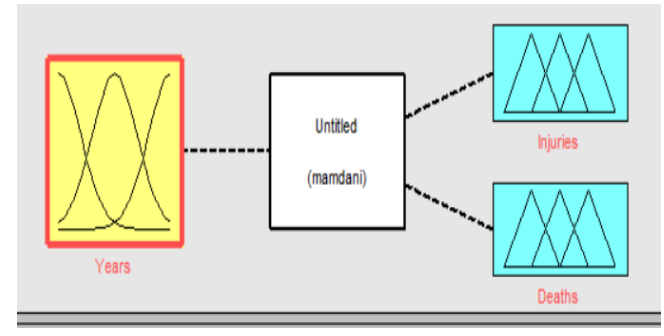

Figure 5: Variables for trends Value

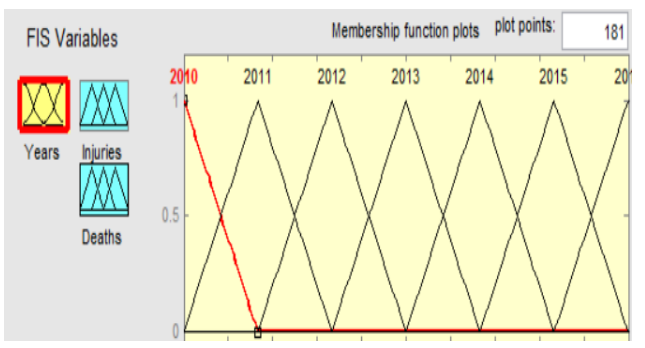

Figure 6: Membership Functions

process of formulating the mapping from a given input to an output using Fuzzy logic. The mapping provides a basis from which decisions can be made. In our agent, FIS involves membership functions, fuzzy logic operators and IF-Then rules. For analyzing the trend value of injuries and deaths year-wise we have taken one 
input variable i.e. year and two output variables i.e. injuries and deaths as shown in the fig.5.

We have taken 7 membership functions for years' variable i.e. from 2010 to 2016, four membership functions for road injuries variable i.e. no, less, average and maximum and similar membership functions for road deaths variable as shown in the fig. 6 . We have shown the trend values of road injuries and from 2010 to 2016 as shown in the fig. 5 and road deaths in fig. 6 .

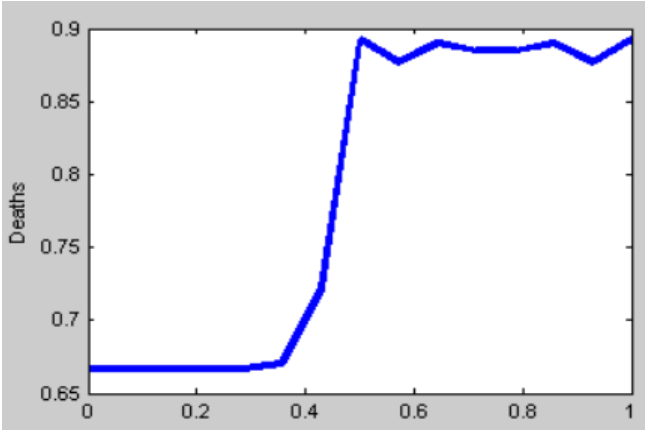

Figure 7: Number of Injuries year-wise

\section{CONCLUSION}

In this paper, we have proposed a cognitive vehicular agent that is able to receive the massive data from clouds, evaluate it and protect itself at run time from road accident, bad weather, near restaurant, near patrol pump on the road at miles using its learning ability. In our proposed cognitive agent, we have used

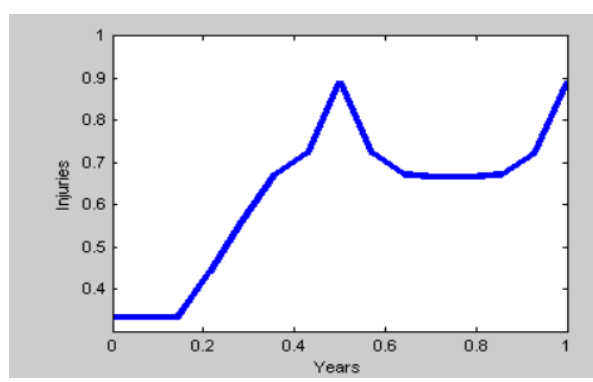

Figure 8: Number of deaths year wise the memories. Each memory its specific function in this agent. Memories are used to receive the massive data from clouds about the weather condition, road condition and its surroundings, restaurants and petrol pumps, store it for taking further action whenever is required in the future automatically. After becoming cognitive agent, it will be able to perform following functions at runtime such as self-integrating, selfconfiguring, self-learning, self-healing, self-driving etc.

\section{FUTURE WORK}

Over the next some decades, vehicles will be so sophisticated to configure themselves to a driver and the world around them automatically. A selfenabled vehicle will be able to take care of its occupants, take care of itself and also work with others in the transportation ecosystem as required. 
MAKING," Interdisciplinary

\section{REFERENCES}

[1] T. Litman, "Autonomous vehicle implementation, predictions," Victoria Transport Policy, 2016.

[2] Heer, "Vehicle to Cloud data transfer standard proposal," EE Online.org.uk, UK, 2016.

[3] H. Christoph, "Toyota Connects navigation systms to the clouds," EE Times, 2013.

[4] G. Mario, "Vehicular Cloud Computing," in 11th Annual Mediterranean Ad Hoc Networking Workshop, Cyprus, 2012`.

[5] P. Alexander, F. Jana and B. Jochen, "Sensory memory of structure from motion is shape specific," Attention, Perception \& Psychophysics, vol. 75, no. 6, pp. 1215-1229, 2013.

[6] M. W. Matlin, Cognition, John Wiley \& Sons, 2005.

[7] R. Lenninger, "Connecting Massive Data, Cloud and Watson to the Car for a Safer Rider Home," Emtech Group, 2015.

[8] K. Gyimesi, "From connected to self-enabling vechicles: what's next in automotive," IBM Big data and Analytics Hub, 2015.

[9] O. Markič, "RATIONALITY AND EMOTIONS IN DECISION
Description of Complex Systems, p. 11, 2009.

[10] M. Pagel, "The Evolution of Human Emotions," Oxford University Press, New York, 2002.

[11] J. Sylvester and N. . V. Chawla, "Evolutionary Ensembles:

Combining Learning Agents using Genetic Algorithms," American Association for Artificial Intelligence, p. 6, 2005.

[12] "Wikipedia-evolution of emotions," 15 march 2012. [Online]. Available: www.wikipedia.com. [Accessed 04 2012].

[13] A. M, L. O. N and A. A. P, "The Effectiveness of Genetic Algorithm in Solving Simultaneous Equations," International Journal of Computer Applications, p. 4, 2011

[14] Athar and K. Ahmed, "Generation of emotions in Neural Networks Based on Experience," in 4th international conference of islamic countries society of statistical sciences, 2008.

[15] M. Kumar, M. Husian, N. Upreti and D. Gupta, "GENETIC ALGORITHM: REVIEWAND APPLICATION," International Journal of Information Technology 
and Knowledge Management, p. 4, 2010.

[16] "positive and negative emotions," [Online]. Available: http://changingminds.org/explanat ions/emotions/emotion_purpo se.htm. [Accessed 04 2012].

[17] "Types of emotions," [Online]. Available: http://www.theemotions.com/type-ofemotions.html. [Accessed 04 2012].

[18] "changingminds.org,"

[Online].

Available:http://changingmin ds.org/explanations/emotions/ emotion_decision.htm.

[Accessed 04 2012]. J. D. Velasquez, "Modeling Emotion-Based decisionMaking," p. 6, 1997. 